\title{
3D Radiative Transfer Equation Coupled with Heat Conduction Equation with Realistic Boundary Conditions Applied on Complex Geometries
}

\author{
D. Le Hardy, Y. Favennec, G. Domingues, B. Rousseau \\ Université de Nantes, Nantes, France \\ Email: david.le-hardy@univ-nantes.fr
}

Received 1 June 2016; accepted 8 August 2016; published 15 August 2016

\begin{abstract}
This paper presents the solution of coupled radiative transfer equation with heat conduction equation in complex three-dimensional geometries. Due to very different time scales for both physics, the radiative problem is considered steady-state but solved at each time iteration of the transient conduction problem. The discrete ordinate method along with the decentered streamline-upwind Petrov-Galerkin method is developed. Since specular reflection is considered on borders, a very accurate algorithm has been developed for calculation of partition ratio coefficients of incident solid angles to the several reflected solid angles. The developed algorithms are tested on a paraboloid-shaped geometry used for example on concentrated solar power technologies.
\end{abstract}

\section{Keywords}

Radiative Transfer Equation, Heat Conduction Equation, Finite Element Methods, SUPG, DOM, Specular Reflection, Complex Geometry

\section{Introduction}

The study of the thermal and radiative heat transfer in semitransparent media plays an important role for industrial applications such as thermal insulation [1], photo-thermal therapy [2], glass forming [3] [4], porous media [5] and many others [6]. The steady thermal equation is commonly used to give a global and sometimes sufficient solution [7] [8] but, in some applications [2] [3], the knowledge of the evolution of the thermal heat transfer is necessary.

The coupling takes into account of the steady-state radiative transfer equation (RTE), as well as the transient heat conduction equation (HCE). Such a transient coupling is well derived in [9] [10]. The RTE is an integro-differential equation that contains an advection term and also an angular integral term corresponding to a gain by scattering. Deterministic and statistical methods are both popular in the radiative transfer community to solve the RTE. For the determinist methods, the most well-known angular discretization methods are the dis- 
crete ordinate methods [9] [11] [12] and the $P_{N}$ methods [2] [10] [13]. The RTE being a hyperbolic equation, the finite volume methods (FVM) are widely used for such kind of equation, for the spatial discretization [14] [15]. To add more, Finite Element Methods (FEM) are useful for complex geometries. In FEM, the classical Galerkin FEM in its original version, is not suited for the RTE, due to first order differential [16]. In such case, the Streamline-Upwind Petrov-Galerkin (SUPG) stabilizes the solution by adding artificial diffusion [11] [17]. Other methods have been developed in the past [9] [10] [18]-[23]. For the statistical methods, the Monte Carlo [23] [24] and Ray Tracing [25] model the transport of photons using samples and randoms. The statistical methods are easy to handle for simple geometries and consume low memory. Moreover, to calculate an exchange between two surfaces/volumes, the methods are fast. However, when the geometries are complex, when the solution is to be found in a whole enclosure, and when the radiative properties are heterogeneous, then the statistical methods are difficult to handle and the CPU time needed to obtain an accurate solution may become extremely high.

Due to the second-order diffusion operator, the Galerkin finite element methods are efficient to solve the HCE as long as the nonlinear partfrom the blackbody radiance is properly dealt with. Lattice Boltzmann [7] and meshless methods [8] may also be good alternative to finite elements for this rather simple physics. Recently, Monte Carlo methods have been developed specifically for coupled conduction, convection and radiative transfers [26].

The paper is organized as follow. In Section 2, the physical models are presented: in one hand the radiative transfer equation along with mixed diffuse/specular boundary conditions, and, in the other hand, the transient heat conduction equation along with its specific boundary conditions. The Section 3 deals with the numerical methods which are used to solve the coupling RTE-HCE. A discrete ordinate method for angular discretization, combined with SUPG, a decentered finite element scheme for space discretization, allow the solution of the RTE. For the HCE, Euler implicit scheme combined with a Galerkin finite element method are used to solve the energy problem. Section 4 finally deals with numerical results. A three-dimensional paraboloid is considered. Numerical results are given for several refractive index coefficients yielding to model on some cases highly reflecting materials as well as, on other cases, non-reflecting boundary reflections. According to cases, the temperature evolution inside the medium of concern greatly changes.

\section{Mathematical Models}

Two models are presented: the radiative transfer equation and the heat transfer equation, both with their respective boundary conditions. The speed of the light being much higher than the conduction time constant, the steady solution of the radiative transfer equation is considered for all given conduction time steps.

The radiative transfer equation is written as follow:

$$
s . \nabla I(x, s)+\beta I(x, s)=\sigma_{s} \int_{4 \pi} \Phi\left(s, s^{\prime}\right) I\left(x, s^{\prime}\right) \mathrm{d} s^{\prime}+\kappa I_{b}(T(t, x))
$$

where $I$ is the radiative intensity for a monochromatic wavelength in $\mathrm{Wm}^{-2} \mu \mathrm{m}^{-1} \mathrm{sr}^{-1}$, solution of the radiative transfer equation which is to be solved for all direction $\boldsymbol{s}$ in the unit sphere and for all $\boldsymbol{x}$ into the open bounded domain D. $\sigma_{s}$ is the scattering coefficient, $\kappa$ is the absorption coefficient and $\beta=\sigma_{s}+\kappa$ is the so-called extinction coefficient in $\mathrm{m}^{-1}, \Phi$ is the scattering phase function and $I_{b}(T)$ is the given Planck function defined for a given wavelength in vacuum $\lambda_{0}$ [9]:

$$
I_{b}(T(t, x))=C_{1} n^{3} \lambda_{0}^{-5}\left(e^{\frac{C_{2}}{\lambda_{0} T}}-1\right)^{-1} \text { in } \mathrm{Wm}^{-2} \mu \mathrm{m}^{-1}
$$

where $C_{1}=1.191 \times 10^{-16} \mathrm{Wm}^{2}, C_{2}=1.4388 \times 10^{-2} \mathrm{Km}$. Moreover, the behavior of the radiative intensity on borders is important to be well taken into account in order to simulate a physics close to the reality. For a smooth media, specular reflection is considered:

$$
I(\boldsymbol{x}, \boldsymbol{s})=\tilde{I}(\boldsymbol{x}, \boldsymbol{s})+\rho(\boldsymbol{s} . \boldsymbol{n}) I(\boldsymbol{x},[\boldsymbol{s}-2(\boldsymbol{s} . \boldsymbol{n}) \boldsymbol{n}]) \text { on } \partial D \text { and } \forall \boldsymbol{s} \text { such as } \boldsymbol{s . n}<0
$$

The first term in the right-hand-side of the equality sign is the Dirichlet contribution which may explain external sources for example. The other term is the gain by specular reflection from the incident direction $\boldsymbol{s}-2($ s.n $) \boldsymbol{n} . \quad \rho(s . n) \in[0,1]$ is the reflection coefficient based on the Fresnel formulation and the Snell-Des- 
cartes law. The reflectivity coefficient depends of the scalar product $\mathbf{s} . \boldsymbol{n}=\cos \theta_{i}$ and the index of refraction $\tilde{n}$.

The blackbody emissivity $I_{b}(T)$ depends explicitly of the temperature $T(t, x)$ supposed unknown. The temperature is the solution of the following unsteady-conduction equation:

$$
\frac{\partial T}{\partial t}(t, \boldsymbol{x})=D_{T} \Delta \mathrm{T}(\mathrm{t}, \mathbf{x})-\nabla \cdot \mathrm{q}_{\mathrm{r}}(t, \boldsymbol{x})
$$

where $D_{T}=\frac{k}{\rho c_{p}}$ is the thermal diffusivity coefficient, $k$ the thermal conductivity $\left(\mathrm{WK}^{-1} \mathrm{~m}^{-1}\right), \rho$ is the density $\left(\mathrm{kgm}^{-3}\right)$ and $c_{p}$ is the specific heat capacity $\left(\mathrm{J} \mathrm{kg}^{-1} \mathrm{~K}^{-1}\right)$. The divergence of the radiative flux $\nabla \cdot q_{r}(t, x)=\kappa I_{b}-\kappa \int_{4 \pi} I(x, s) \mathrm{d} s$ depends on the radiative intensity and the temperature. The temperature at $t=0$ is supposed known $T(0, \boldsymbol{x})=T_{0}(\boldsymbol{x})$. Also, Robin boundary conditions areapplied on borders to simulate convective transfers with an external fluid at temperature $T_{\text {ext }}$ :

$$
\frac{\partial T}{\partial \boldsymbol{n}}(t, \boldsymbol{x})=h\left(T_{\text {ext }}(\boldsymbol{x})-T(t, \boldsymbol{x})\right) \text { on } \partial D
$$

where $h$ is the exchange coefficient.

\section{Mathematical Approximations}

In the general case, the RTE and the HCE cannot be solved analytically. Some numerical tools need to be developed to get an approximation of the continuous solution. The discretization of the RTE and the HCE are respectively presented.

To cut off the integral problem into the radiative transfer equation, the unit sphere is discretized into $N_{d}$ solid angles with a main direction $s_{m}$. The radiative transfer equation becomes a system of $N_{d}$ equations with $N_{d}$ unknowns, noted $I_{m}(\boldsymbol{x})$ each being continuous in space. As the number of directionsis limited, the specular condition is also discretized accordingly:

$$
\begin{gathered}
\boldsymbol{s}_{\boldsymbol{m}} \cdot \nabla I_{m}(\boldsymbol{x})+\beta I_{m}(\boldsymbol{x})=\sigma_{s} \sum_{j=1}^{N_{d}} \omega_{j} \Phi_{m, j} I_{j}(\boldsymbol{x})+\kappa I_{b}(T), \forall m=1, \ldots, N_{d} \\
I_{m}(\boldsymbol{x})=\tilde{I}_{m}(\boldsymbol{x})+\rho\left(\boldsymbol{s}_{\boldsymbol{m}} \cdot \boldsymbol{n}\right) \sum_{s_{j} \cdot \boldsymbol{n}>0} \delta_{m, j}(\boldsymbol{n}) I_{j}(\boldsymbol{x})
\end{gathered}
$$

where $\omega_{j}$ corresponds to the weight associated to the direction $\boldsymbol{s}_{j}$, and $\delta_{m, j}(\boldsymbol{n})$ is the partition ratio coefficient representing the proportion of the radiative intensity $I_{j}$ which is reflected towards the direction $s_{m}$, taking into account of the weight $\rho\left(\boldsymbol{s}_{\boldsymbol{m}} \cdot \boldsymbol{n}\right)$ according to Fresnel law.

The Galerkin finite element method being as well known unstable for the radiative transfer equation due to the advection term $s . \nabla I_{m}$, the streamline-upwind Petrov-Galerkin method uses an additional term to the test function $v$ to throw off the scheme and to get stability. To obtain the weak formulation, the $m^{\text {th }}$ equation of the global system is multiplied by the test function $v+\gamma s_{m} . \nabla v$, it is then integrated on the full domain $D$, and the Green theorem is finally used to express the boundary conditions. To add stability, $\gamma=0.3 h_{D}$, with $h_{D}$ depend of the mesh refinement of $D$ [17]. The variational formulation reads, with $\tilde{\beta}_{m}=\kappa+\sigma_{s}\left(1-\omega_{m} \Phi_{m, m}\right)$ :

$$
\begin{aligned}
& \int_{D}\left[I_{m}-\gamma\left(\boldsymbol{s}_{\boldsymbol{m}} \cdot \nabla I_{m}\right)\right]\left[\tilde{\beta}_{m} v-\boldsymbol{s}_{\boldsymbol{m}} \cdot \nabla v\right] \mathrm{d} \boldsymbol{x}+\int_{s_{m} \cdot \boldsymbol{n}>0}\left(1+\tilde{\beta}_{m} \gamma\right) I_{m} v \boldsymbol{s}_{\boldsymbol{m}} \cdot \boldsymbol{n} \mathrm{d} \Gamma \\
& -\sum_{j \neq m} \omega_{j} \Phi_{m, j} \int_{D} \sigma_{s} I_{j}\left(v+\gamma \boldsymbol{s}_{\boldsymbol{m}} \cdot \nabla v\right) \mathrm{d} \boldsymbol{x}+\rho\left(\boldsymbol{s}_{\boldsymbol{m}}, \boldsymbol{n}\right) \int_{s_{i} \cdot \boldsymbol{n}>0 \cap s_{\boldsymbol{m}_{m} \cdot n<0} \delta_{m j}(\boldsymbol{n}) I_{j} \boldsymbol{v n} \cdot \boldsymbol{s}_{\boldsymbol{m}} \mathrm{d} \Gamma} \\
& =\int_{s_{\boldsymbol{m}} \cdot \boldsymbol{n}>0} \tilde{I}_{m} v \boldsymbol{s}_{\boldsymbol{m}} \cdot \boldsymbol{n} \mathrm{d} \Gamma+\int_{D} I_{b}\left(v+\gamma \boldsymbol{s}_{\boldsymbol{m}} \cdot \nabla v\right) \mathrm{d} \boldsymbol{x}
\end{aligned}
$$

To cut off the temporal derivative, the first order implicit Euler scheme is used. Moreover, at a given time step, the divergence of the flux $q_{r}$ is calculated at the previous time step to remove the nonlinearity due to the blackbody term $I_{b}(T)$. The weak formulation of the conduction problem reads:

$$
\int_{D} \frac{T^{N+1}}{\delta t}+D_{T} \nabla T^{N+1} \cdot \nabla v \mathrm{~d} \boldsymbol{x}+\int_{\partial D} \frac{D h}{k} T^{N+1} v \mathrm{~d} \Gamma=\int_{D} \frac{T^{N}}{\delta t} v-\nabla \cdot q_{r}^{N} v \mathrm{~d} \boldsymbol{x}+\int_{\partial D} \frac{D h}{k} T_{\text {ext }} v \mathrm{~d} \Gamma
$$




\section{Numerical Solution}

The set of the varatiational Formulations (9)-(11) gives us steady-state solutions of radiative intensities along with the transient solution of the temperature in the whole domain. The geometry of concern is a paraboloid with a height of $4 / 3 \mathrm{~m}$ and a diameter of $4 \mathrm{~m}$. The equation of the paraboloid surface is given by $z=0.333\left(x^{2}+y^{2}\right)$. The physical properties are the following. The absorption coefficient is $\kappa=0.4 \mathrm{~m}^{-1}$, the isotropic scattering coefficient is $\sigma_{s}=0.1 \mathrm{~m}^{-1}$. Next, the thermal conductivity is $k=1 \mathrm{Wm}^{-1} \mathrm{~K}^{-1}$, the density is $\rho=2 \mathrm{kgm}^{-3}$, the heat capacity is $c_{p}=5 \mathrm{~J} \mathrm{~kg}^{-1} \mathrm{~K}^{-1}$, and the convective exchange coefficient is $h=5 \mathrm{~m}^{-2}$. At $t=0 \mathrm{~s}$, the temperature is $T_{0}(\boldsymbol{x})=300 \mathrm{~K}$. A collimated beam is entering to the medium on the full plan surface such as $I\left(\boldsymbol{x}, \boldsymbol{s}_{0}\right)=10^{-3} \mathrm{Wm}^{-2} \mu \mathrm{m}^{-1} \mathrm{sr}^{-1}$. Solutions are presented below for three values of refractive index. The first case, with $n=1$, considers the border is transparent, there is no reflection. Another case, with $n=1.8$, considers that the reflected part is very important. The last case stands in between, with $n=1.4$.

Figure 1 presents the evolutions of the radiative intensity and of temperature at $t=0.5 \mathrm{~s}$ along the longitudinal axis, and Figure 2 presents the same data in cross-sections. It can be observed that the maximum radiative intensity increases with the refraction index. Hence worth, the temperature inside the medium also greatly in-

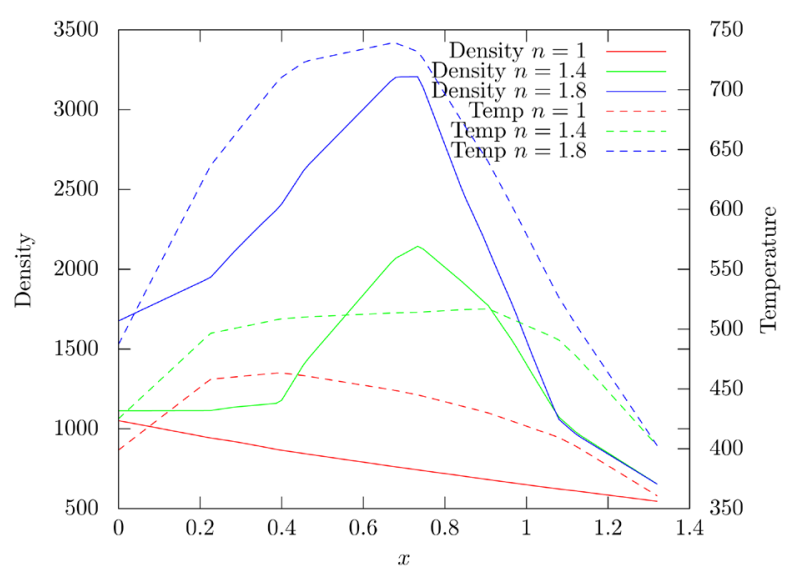

Figure 1. Evolution of the radiative intensity and of temperature à $\mathrm{t}=0.5 \mathrm{~s}$ along the longitudinal axis.

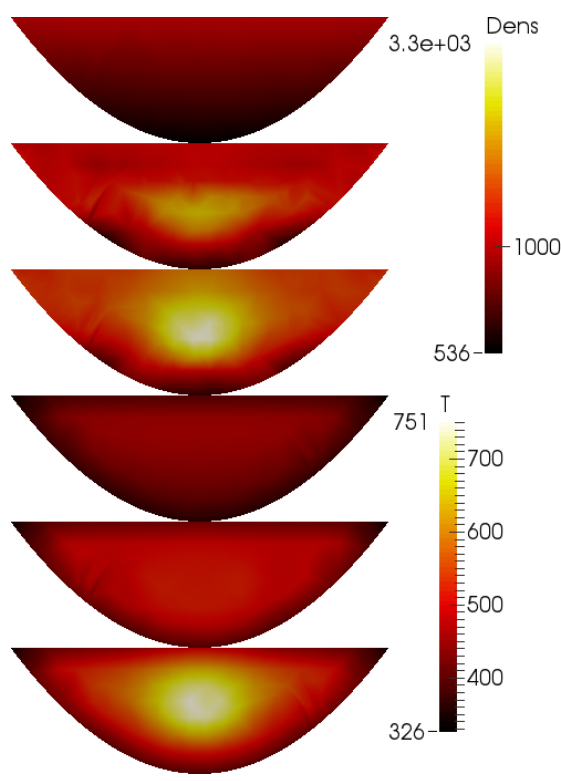

Figure 2. Top: radiative intensity; bottom: temperature at $t=0.5 \mathrm{~s}$. For each, the first is for $n=1$, the second is for $\mathrm{n}=1.4$, the third is for $\mathrm{n}=1.8$. 
creases with the refraction index. As an example an increase of the index factor from 1 to 1.4 increases the maximum temperature difference from 164 to $220 \mathrm{~K}$. In the same manner, an increase of the index factor from 1.4 to 1.8 increases the maximum temperature difference from 220 to $551 \mathrm{~K}$. This confirms that the design of materials for such systems is highly important.

\section{References}

[1] Asllanaj, F., Jeandel, G. and Roche, J.R. (2001) Numerical Solution of Radiative Transfer Equation Coupled with Nonlinear Heat Conduction Equation. International Journal of Numerical Methods for Heat \& Fluid Flow, 11, 449473. http://dx.doi.org/10.1108/EUM0000000005528

[2] Bruno, A.B., et al. (2016) Numerical Simulation of Nanoparticles Assisted Laser Photothermal Therapy: A Comparison of the P1-Approximation and Discrete Ordinate Methods. Journal of the Brazilian Society of Mechanical Sciences and Engineering, 1-10. http://dx.doi.org/10.1007/s40430-016-0553-3

[3] Lacroix, D., et al. (2002) Coupled Radiative and Conductive Heat Transfer in a Non-Grey Absorbing and Emitting Semitransparent Media under Collimated Radiation. Journal of Quantitative Spectroscopy and Radiative Transfer, 75, 589-609. http://dx.doi.org/10.1016/S0022-4073(02)00031-6

[4] David, L., et al. (2006) Transient Radiative and Conductive Heat Transfer in Non-Gray Semitransparent Two-Dimensional Media with Mixed Boundary Conditions. Heat and Mass Transfer, 42, 322-337. http://dx.doi.org/10.1007/s00231-005-0023-4

[5] Li, G.-J., Ma, J. and Li, B.-W. (2015) Collocation Spectral Method for the Transient Conduction-Radiation Heat Transfer with Variable Thermal Conductivity in Two-Dimensional Rectangular Enclosure. Journal of Heat Transfer, 137, 032701.

[6] Luo, X.-H., et al. (2014) Simulation of Thermal Radiation Effects on MHD Free Convection in a Square Cavity Using the Chebyshev Collocation Spectral Method. Numerical Heat Transfer, Part A: Applications, 66, 792-815. http://dx.doi.org/10.1080/10407782.2013.873293

[7] Sun, J., Yi, H.-L. and Tan, H.-P. (2016) Local RBF Meshless Scheme for Coupled Radiative and Conductive Heat Transfer. Numerical Heat Transfer, Part A: Applications, 1-15.

[8] Wang, C.-A., Sadat, H. and Tan, J.-Y. (2016) Meshless Method for Solving Coupled Radiative and Conductive Heat Transfer in Refractive Index Medium. Journal of Physics: Conference Series, 676, IOP Publishing.

[9] Howell, J.R., Pinar Menguc, M. and Siegel, R. (2010) Thermal Radiation Heat Transfer. CRC Press.

[10] Modest, M.F. (2003) Radiative Heat Transfer. 2nd Edition, Academic Press, San Diego.

[11] Le Hardy, D., Favennec, Y. and Rousseau, B. (2016) Solution of the 2-D Steady-State Radiative Transfer Equation in Participating Media with Specular Reflections Using SUPG and DG Finite Elements. Journal of Quantitative Spectroscopy and Radiative Transfer, 179, 149-164. http://dx.doi.org/10.1016/j.jqsrt.2016.03.027

[12] Castro, R.O. and Trelles, J.P. (2015) Spatial and Angular Finite Element Method for Radiative Transfer in Participating Media. Journal of Quantitative Spectroscopy and Radiative Transfer, 157, 81-105. http://dx.doi.org/10.1016/j.jqsrt.2015.02.008

[13] Hachem, E., et al. (2012) Immersed Volume Method for Solving Natural Convection, Conduction and Radiation of a Hat-Shaped Disk inside a 3D Enclosure. International Journal of Numerical Methods for Heat \& Fluid Flow, 22, 718741. http://dx.doi.org/10.1108/09615531211244871

[14] Boulet, P., Collin, A. and Consalvi, J. (2007) On the Finite Volume Method and the Discrete Ordinates Method Regarding Radiative Heat Transfer in Acute Forward Anisotropic Scattering Media. Journal of Quantitative Spectroscopy and Radiative Transfer, 104, 460-473. http://dx.doi.org/10.1016/j.jqsrt.2006.09.010

[15] Coelho, P.J. (2014) Advances in the Discrete Ordinates and Finite Volume Methods for the Solution of Radiative Heat Transfer Problems in Participating Media. Journal of Quantitative Spectroscopy and Radiative Transfer, 145, 121-146. http://dx.doi.org/10.1016/j.jqsrt.2014.04.021

[16] Zhang, L., Zhao, J.M. and Liu, L.H. (2009) Finite Element Method for Modeling Radiative Transfer in Semitransparent Graded Index Cylindrical Medium. Journal of Quantitative Spectroscopy and Radiative Transfer, 110, 1085-1096. http://dx.doi.org/10.1016/j.jqsrt.2009.03.018

[17] Kanschat, G., Meinköhn, E., Rannacher, R. and Wehrse, R. (2009) Numerical Methods in Multidimensional Radiative Transfer. Springer. http://dx.doi.org/10.1007/978-3-540-85369-5

[18] Sun, Y.-S. and Li, B.-W. (2010) Spectral Collocation Method for Transient Conduction-Radiation Heat Transfer. Journal of Thermophysics and Heat Transfer, 24, 823-832. http://dx.doi.org/10.2514/1.43400

[19] Sun, Y.J. and Zhang, X.B. (2016) Analysis of Transient Conduction and Radiation Problems Using Lattice Boltzmann and Finite Volume Methods. International Journal of Heat and Mass Transfer, 97, 611-617. 
http://dx.doi.org/10.1016/j.ijheatmasstransfer.2016.01.074

[20] Zmywaczyk, J. and Koniorczyk, P. (2009) Numerical Solution of Inverse Radiative-Conductive Transient Heat Transfer Problem in a Grey Participating Medium. International Journal of Thermophysics, 30, 1438-1451. http://dx.doi.org/10.1007/s10765-009-0635-X

[21] Sakurai, A., Mishra, S.C. and Maruyama, S. (2010) Radiation Element Method Coupled with the Lattice Boltzmann Method Applied to the Analysis of Transient Conduction and Radiation Heat Transfer Problem with Heat Generation in a Participating Medium. Numerical Heat Transfer, Part A: Applications, 57, 346-368. http://dx.doi.org/10.1080/10407780903583008

[22] Tencer, J. and Howell, J.R. (2015) Coupling Radiative Heat Transfer in Participating Media with Other Heat Transfer Modes. Journal of the Brazilian Society of Mechanical Sciences and Engineering, 1-15.

[23] Cherkaoui, M., Dufresne, J.-L., Fournier, R., Grandpeix, J.-Y. and Lahellec, A. (1996) Monte Carlo Simulation of Radiation in Gases with a Narrow-Band Model and a Net-Exchange Formulation. Journal of Heat Transfer, 118, 401407. http://dx.doi.org/10.1115/1.2825858

[24] Delatorre, J., et al. (2014) Monte Carlo Advances and Concentrated Solar Applications. Solar Energy, 103, $653-681$. http://dx.doi.org/10.1016/j.solener.2013.02.035

[25] Kajiya, J.T. and Von Herzen, B.P. (1984) Ray Tracing Volume Densities. ACM SIGGRAPH Computer Graphics, 18. http://dx.doi.org/10.1145/800031.808594

[26] Fournier, R., et al. (2016) Radiative, Conductive and Convective Heat-Transfers in a Single Monte Carlo Algorithm. Journal of Physics: Conference Series, 676, IOP Publishing.

\section{Submit or recommend next manuscript to SCIRP and we will provide best service for you:}

Accepting pre-submission inquiries through Email, Facebook, LinkedIn, Twitter, etc. A wide selection of journals (inclusive of 9 subjects, more than 200 journals)

Providing 24-hour high-quality service

User-friendly online submission system

Fair and swift peer-review system

Efficient typesetting and proofreading procedure

Display of the result of downloads and visits, as well as the number of cited articles

Maximum dissemination of your research work

Submit your manuscript at: http://papersubmission.scirp.org/ 